\title{
Ginsenoside Rg1 improves pathological damages by activating the p21-p53-STK pathway in ovary and Bax-Bcl2 in the uterus in premature ovarian insufficiency mouse models
}

\author{
LIANLI HE ${ }^{1}$, XIAOJUAN WANG ${ }^{1}$, DAIGANG CHENG $^{1}$, ZHENGAI XIONG $^{2}$ and XIAOYUN LIU ${ }^{1}$ \\ ${ }^{1}$ Department of Gynecology and Obstetrics, The First People's Hospital of Zunyi and Third Affiliated Hospital \\ of Zunyi Medical University, Zunyi, Guizhou 563000; ${ }^{2}$ Department of Gynecology and Obstetrics, \\ The Second Affiliated Hospital, Chongqing Medical University, Chongqing 400010, P.R. China
}

Received June 20, 2019; Accepted August 25, 2020

DOI: $10.3892 / \mathrm{mmr} .2020 .11675$

\begin{abstract}
The aim of the present study was to investigate the effects of the ginsenoside Rg1 on D-galactose (D-gal)-induced mouse models of premature ovarian insufficiency (POI) and the related mechanisms. C57BL/6 female mice were randomly grouped into the following: i) D-gal [subcutaneously (s.c.) $200 \mathrm{mg} / \mathrm{kg} / \mathrm{d}$ D-gal for 42 days]; ii) $\operatorname{Rg} 1$ [intraperitoneally (i.p.) $20 \mathrm{mg} / \mathrm{kg} / \mathrm{d} \operatorname{Rg} 1$ for 28 days]; iii) D-gal + Rg1 (s.c. $200 \mathrm{mg} / \mathrm{kg} / \mathrm{d}$ D-gal for 42 days followed by i.p. $20 \mathrm{mg} / \mathrm{kg} / \mathrm{d} \mathrm{Rg} 1$ for 28 days); and iv) saline groups (equivalent volume of saline s.c. and i.p.). Hematoxylin and eosin staining and electron microscopy were used to analyze uterine and ovarian morphology. Expression levels of senescence factors (p21, p53 and serine/threonine kinase), secretion of pro-inflammatory cytokines [interleukin (IL)-6, tumor necrosis factor (TNF)- $\alpha$ and IL-1 $\beta$ ] and the activities of oxidation biomarkers [superoxide dismutase (T-SOD), malondialdehyde (MDA) and glutathione peroxidase (GSH-px)] were analyzed. The results showed that mice in the $\operatorname{Rg} 1+\mathrm{D}$-gal group had significantly higher uterine and ovarian weight compared with those in the D-gal group. Uterus morphology was also improved, based on the comparison
\end{abstract}

Correspondence to: Professor Zhengai Xiong, Department of Gynecology and Obstetrics, The Second Affiliated Hospital, Chongqing Medical University, 9 Linjiang Road, Chongqing 400010, P.R. China

E-mail: docxza1@163.com

Professor Xiaoyun Liu, Department of Gynecology and Obstetrics, The First People's Hospital of Zunyi and Third Affiliated Hospital of Zunyi Medical University, 98 Fenghuang Road, Zunyi, Guizhou 563000, P.R. China

E-mail: 656479556@qq.com

Abbreviations: D-gal, D-galactose; POI, premature ovarian insufficiency

Key words: premature ovarian insufficiency, ginsenoside $\mathrm{Rg} 1$, ovary, uterus between the D-gal group and the $\mathrm{Rg} 1+\mathrm{D}$-gal group. In addition, the $\operatorname{Rg} 1$ treatment after D-gal administration significantly decreased the expression of senescence-associated factors, enhanced the activities of anti-oxidant enzymes total T-SOD and GSH-px in addition to reducing TNF- $\alpha$, IL-1 $\beta$, MDA and IL-6 (based on the comparison between the D-gal group and the Rg1 + D-gal group). In conclusion, the present study suggested that the ginsenoside Rg1 improved pathological damages in the ovary and uterus by increasing anti-oxidant and anti-inflammatory abilities whilst reducing the expression of senescence signaling pathways in POI mouse models.

\section{Introduction}

In recent years, due to the environmental pollution, as well as increasing living and working pressure, premature ovarian insufficiency (POI) and female infertility have become a global issue $(1,2)$. POI is defined as the cessation of ovarian function, which usually occurs before the age of 40 and is characterized by reduced estrogen levels and elevated gonadotropin levels (3). It has been previously reported that the incidence of POI in females $\leq 40$ years of age is $\sim 1 \%$ whereas incidence for females aged $<30$ years is $1(4,5)$. In addition, among female patients with POI, 10-28\% would experience primary amenorrhea whilst $4-18 \%$ would exhibit secondary amenorrhea $(4,5)$.

The pathogenesis of POI remains poorly understood (6). At present, follicle donation and hormone replacement therapy (HRT) are the primary treatment methods for POI (7). In particular HRT can also relieve the symptoms of POI. However, the clinical application of HRT is limited due to the increased risk of endometrial cancer, breast cancer, vaginal bleeding, liver and kidney function impairment and vascular embolism (8). Therefore, it would be of great value to identify novel agents that confer little to no side effects but can significantly improve the symptoms in a similar manner to HRT. The ginsenoside Rg1 is a natural estrogen that has various reported pharmacological anti-oxidation and anti-aging effects $(9,10)$. Previous studies (11-13) demonstrated that unlike synthetic estrogen, $\operatorname{Rg} 1$ would not increase the risk of breast cancer and endometrial cancer (14-16). Mitochondrial 
oxidative stress can be treated by enhancing endogenous antioxidants $(17,18)$. However, it remains unknown whether senescence of the reproductive system can be delayed by Rg1 by enhancing the anti-oxidation pathway in a D-galactose (D-gal)-induced rodent aging model.

D-gal is an agent that has been widely recognized to induce aging and has been previously used for the establishment of animal models of aging in various organs $(19,20)$. To date, $\sim 400$ articles have been reported using this model. It has also been reported that female individuals (fish, fruit flies, rats and mice) with galactosemia would eventually develop POI (21). The mechanisms underlying POI would likely be revealed by investigating individuals with galactosemia (22). Continuous injection of D-gal elevates galactose levels, leading to the further accumulation of free radicals and subsequent oxidative damage, resulting in the acceleration of the aging process of cells (20). The mechanism of this type of aging process is similar to that of natural aging (23). Additionally, a $\mathrm{D}$-gal-induced aging model is easy to establish (24). Therefore, a D-gal-induced aging model can be regarded to be an adequate model for the investigation into the mechanisms underlying ovary aging.

The p53-p21-serine/threonine kinase (STK) pathway is an important signal transduction pathway involved in the aging process (25). This signaling pathway is associated with the p53-mediated DNA stress damage and repairing (26). The P53 gene is a tumor suppressor gene that encodes the $\mathrm{p} 53$ protein and serves a decisive role in repairing stress-induced DNA damage and the maintenance of gene and genome stability (27). P53 expression is low in normal cells, but can be activated when cells are under stress in conditions such as DNA repair and apoptosis (28). In turn, the p53-encoded product can regulate Bax expression, which may mediate $\mathrm{p} 53$-dependent apoptosis. In addition, p53 can indirectly induce apoptosis by downregulating Bcl2 expression (29). Therefore, apoptosis and aging are processes that are mutually associated. The detection of STK, p21, p53, Bcl2 and Bax of the apoptosis signaling pathway may be useful in understanding the possible mechanisms of D-gal-induced uterine aging.

In the present study, an aging model was established in mice by $\mathrm{D}$-gal treatment, following which the effects of $\mathrm{Rg} 1$ on the histopathology of the ovary and uterus were investigated. The weights of the uterus and ovary, and the expression of oxidative stress and inflammatory biomarkers in the uterus and ovary were then measured and analyzed.

\section{Materials and methods}

Study animals. In total, 100 C57BL/6 female mice (SPF grade), 6-8-weeks old, weighing $20 \pm 2 \mathrm{~g}$ (ranging from 18 to $20 \mathrm{~g}$ ) were obtained from the Medical and Laboratory Animal Center of Chongqing (Chongqing, China). These animals were housed in standard conditions, in a 12/12 h dark/light cycle, at $18-20^{\circ} \mathrm{C}$, with free access to food and water. All animal experimental procedures were approved by the Institutional Animal Care and Use Committee of Chongqing Medical University. All surgeries were performed under sodium pentobarbital anesthesia. Reduced heart rate and decreased respiration rate were used as the main humane endpoints to determine when animals should be euthanized. 'Guidelines for euthanasia of experimental animals' were followed to minimize suffering and distress of animals (30).

The mice were subsequently divided into the following four groups randomly ( $\mathrm{n}=25$ mice per group): (i) The $\mathrm{D}$-gal group, where the mice were subcutaneously (s.c.) injected with D-gal $(200 \mathrm{mg} / \mathrm{kg} / \mathrm{d}$ for 42 days) and intraperitoneally (i.p.) injected with an equivalent volume $(2 \mathrm{ml})$ of saline (daily from day 15 onwards for 28 days); ii) the $\mathrm{Rg} 1$ group, where the mice were first s.c. injected with an equivalent volume $(2 \mathrm{ml})$ of saline (daily for 14 days), followed by an i.p. injection of $20 \mathrm{mg} / \mathrm{kg} / \mathrm{d}$ ginsenoside $\mathrm{Rg} 1$ daily for 28 days (cat. no. RSZD-121106; purity, 98.3\%; dissolved in $\mathrm{ddH}_{2} \mathrm{O}$; Xi'an Haoxuan Biological Technology Co., Ltd.); iii) the $\operatorname{Rg} 1+$ D-gal group, where the mice were first s.c. injected with only D-gal $(200 \mathrm{mg} / \mathrm{kg} / \mathrm{d}$ daily for 42 days), followed by a combination treatment with i.p. $\operatorname{Rg} 1$ injection $(20 \mathrm{mg} / \mathrm{kg} / \mathrm{d}$, daily), for another 28 days); and iv) the saline group (negative control), where the mice received equivalent volume of saline (s.c. and i.p.) at the same time points.

After 42 days of injection, the mice were anaesthetized with $2-3 \%$ isoflurane in a specialized chamber $(31,32)$. When the limbs of the mice were paralyzed and their breathing slowed down, they were taken out of the inducing chamber. The corneal and pain reflexes of mice were then examined. If both corneal and pain reflexes were absent, the mice were considered to be under full anesthesia. Once anaesthetized, their eyeballs were quickly removed and whole blood samples were collected, following which the mice were sacrificed by hemorrhagic shock. Animal death was confirmed by observing cardiac and respiratory arrest for 3-5 min before the ovaries and uterus tissues were removed and weighed.

Microscopy preparation. For light microscopy, specimens were fixed in $10 \%$ neutral formalin (at room temperature for $24 \mathrm{~h}$ ) and embedded with paraffin. The specimens were sectioned (3-4 $\mu \mathrm{m})$, which were then subjected to hematoxylin and eosin (H\&E), Gomori's trichrome and van Gieson elastic staining.

For electron microscopy, specimens were promptly fixed in $4 \%$ glutaraldehyde with $0.1 \mathrm{M}$ cacodylate buffer $\left(\right.$ at $4^{\circ} \mathrm{C}$ for $>2 \mathrm{~h}$ ) and fixed in $1.0 \%$ osmium tetroxide (at $4^{\circ} \mathrm{C}$ for $1 \mathrm{~h}$ ). After dehydration using an ascending ethanol gradient followed by propylene oxide, the specimens were embedded in Epon 812 (Beijing XinWangWeiTuo Technology Co., Ltd.). After sectioning, the specimens were cut into ultra-thin sections using a Porter-Blum MT-2 ultramicrotome and stained with uranyl acetate and lead citrate (at $60^{\circ} \mathrm{C}$ for $24 \mathrm{~h}$ ). These sections were observed under a Hitachi HU-11D electron scanning microscope.

$H \& E$ staining. On the last day of drug administration (day 42), animals were sacrificed under anesthesia and the uterus and ovary tissues were removed from each animal. The tissues were fixed with $4 \%$ paraformaldehyde at $4^{\circ} \mathrm{C}$ overnight, dehydrated using an ascending ethanol gradient followed by xylene and embedded in paraffin. The embedded tissues were then cut into $5-\mu \mathrm{m}$ thick continuous sections, which were stained with $\mathrm{H} \& \mathrm{E}$ (at $4^{\circ} \mathrm{C}$ for $24 \mathrm{~h}$ ). The number of ovarian follicles was analyzed with a light microscope using the Image-Pro Plus 6.0 software (Media Cybernetics, Inc.). 

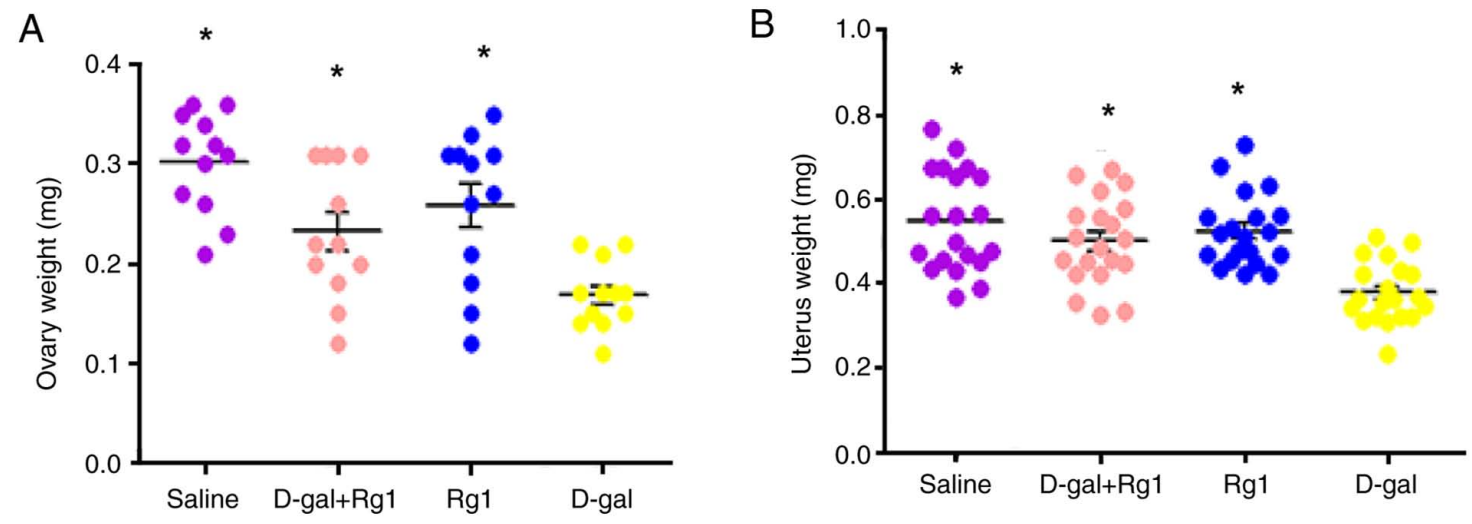

Figure 1. Effects of $\operatorname{Rg} 1$ treatment on the weight of ovary and uterus tissues. Effects of $\operatorname{Rg} 1$ on (A) ovary and (B) uterus weights were evaluated. ${ }^{*} \mathrm{P}<0.05$ vs. D-gal. Rg1, ginsenoside Rg1; D-gal, D-galactose.

Chemical colorimetric analysis. On day 42, ovary and uterus tissues were collected and lysed with RIPA lysis buffer (cat.no.P0013B;BeyotimeInstitute of Biotechnology)for $30 \mathrm{~min}$ on ice. After centrifugation at $10,000 \mathrm{x}$ g at $4^{\circ} \mathrm{C}$ for $10 \mathrm{~min}$, the supernatant was collected. The activities or contents of oxidation-associated biomarkers superoxide dismutase (T-SOD; cat. no. A001-3), malondialdehyde (MDA; cat. no. A003-1) and glutathione peroxidase (GSH-px; cat. no. A005) were measured using corresponding chemical colorimetric assay kits (Nanjing Jiancheng Bioengineering Institute).

ELISA. The levels of interleukin (IL)-6 (cat. no. EK0411), tumor necrosis factor (TNF)- $\alpha$ (cat. no. EK0527) and IL-1 $\beta$ (cat. no. EK0394; all purchased from Wuhan Boster Biological Technology, Ltd.) in tissue lysates of the ovary and uterus were measured using corresponding ELISA kits in accordance with the manufacturer's protocols. In addition, the contents of estradiol 2 (E2; cat. no. EK7003), follicle-stimulating hormone (FSH; cat. no. EK0302) and anti-mullerian hormone (AMH; cat. no. EK1037) were also detected using the corresponding kits (Wuhan Boster Biological Technology, Ltd.) according to the manufacturer's protocols.

Reverse transcription-quantitative PCR (RT-qPCR). Ovary and uterus tissues were first harvested before total RNA was extracted using the TRIzol ${ }^{\circledR}$ reagent (Biolab Biotechnology Co., Ltd.). ReverTra Ace- $\alpha^{\mathrm{TM}}$ first strand cDNA synthesis kit (Toyobo Life Science) was used for reverse transcription (denaturation at $94^{\circ} \mathrm{C}$ and annealing at $55^{\circ} \mathrm{C}$ ). qPCR was subsequently performed using the CFX96 Real-Time PCR detection kit (Biolab Biotechnology Co., Ltd.) was used with the following conditions: Initial denaturation at $94^{\circ} \mathrm{C}$ for $5 \mathrm{~min}$, followed by 35 cycles of denaturation at $94^{\circ} \mathrm{C}$ for $20 \mathrm{sec}$, annealing at $55^{\circ} \mathrm{C}$ for $30 \mathrm{sec}$, and extension at $72^{\circ} \mathrm{C}$ for $30 \mathrm{sec}$. The primer sequences were as follows: p21 forward, 5'-AGTGTGCCGTTGTCTCTTCG-3' and reverse, 5'-ACA CCAGAGTGCAAGACAGC-3'; p53 forward, 5'-AGAGAC CGCCGTACAGAAGA-3' and reverse, 5'-CTGTAGCAT GGGCATCCTTT-3'; STK forward, 5'-GTCGCAGGTTCT TGGTCACT-3' and reverse, 5'-CGAATCTGCACCGTAGTT GA-3'; Bax forward, 5'-AAACTGGTGCTCAAGGCCCT-3' and reverse, 5'-AGCAGCCGCTCACGGAG-3'; Bcl-2 forward, 5'-AGCGACGAGAGA AGTCATCC-3' and reverse, 5'-CTG
TAGCATGGGCATCCTTT-3'; and GAPDH forward, 5'-GCA AAGTGGAGATTGTTGCC-3' and reverse, 5'-CCGTATTCA TTGTCATACCA-3'. The $2^{-\Delta \Delta C q}$ method was used to calculate relative expression levels of target genes (33).

Statistical analysis. Data were presented as the mean \pm SD. Statistical analysis was performed with SPSS 17.0 software (SPSS, Inc.). One-way ANOVA was performed for group comparisons followed by Tukey's test. $\mathrm{P}<0.05$ was considered to indicate a statistically significant difference.

\section{Results}

Rg1 increases the weight coefficient of ovary and uterus. To investigate the effects of Rg1 on the mouse POI model, the weights of ovary and uterus were analyzed after D-gal and/or Rg1 administration. D-gal treatment significantly reduced the ovary and uterus weight compared with that in the saline group (Fig. 1). In addition, the weights of these two organs in the Rg1 + D-gal group exhibited increases compared with those in the D-gal group, suggesting that the inhibitory effects of D-gal were reversed by the injection of Rg1. However, no significant difference was found between the $\operatorname{Rg} 1$ and saline groups (Fig. 1). Therefore, these data suggest that Rg1 can improve the general condition of POI model mice.

Rg1 improves pathological damages in the ovary and uterus. Pathological changes in the ovary and uterus were evaluated by the H\&E staining (Fig. 2) and electron microscopy (Fig. 3). As shown in Fig. 2A, the pathological changes in uterine muscles induced by D-gal were improved by $\operatorname{Rg} 1$ treatment. Statistically, the endometrial thickness of the $\operatorname{Rg} 1$ group was significantly higher than the $\mathrm{D}$-gal group $(\mathrm{P}<0.05$; Fig. 2B). Electron microscopy showed that large quantities of myeloid bodies could be observed in the granule cells with nuclear brittle fissure, nuclear dissolution and nucleation in the granule cells in uterus tissues from the D-gal group (Fig. 3). Additionally, there was necrotic tissue in the ovarian stroma in the D-gal group. However, none were observed in tissues from the Rg1, Rg1 + D-gal or saline groups. There were also large numbers of myeloid bodies and fibrosis in the uterine muscle from the D-gal group, which were found to be improved in that of Rg1, Rg1 + D-gal and saline groups. These results suggest 
A

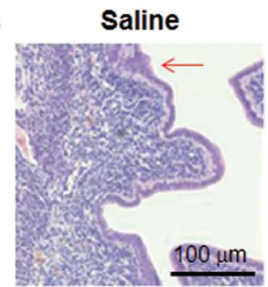

Rg1

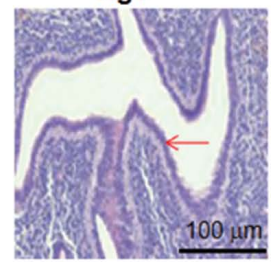

D-gal+Rg1

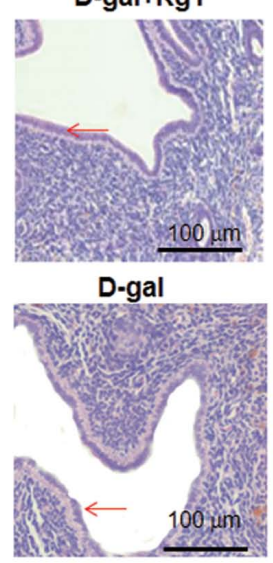

B

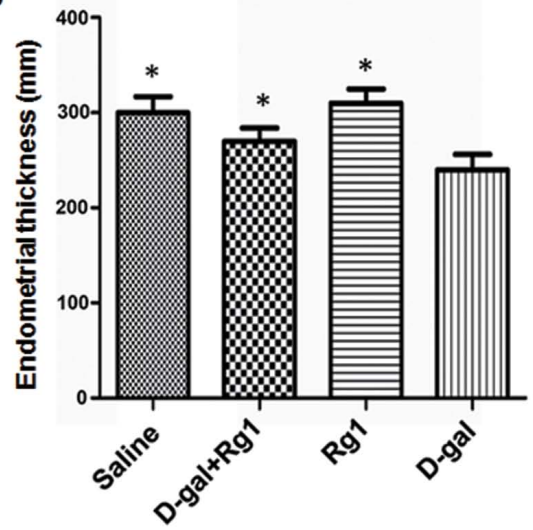

Figure 2. Rg1 treatment reduces reproductive uterus injuries in POI mice. (A) H\&E staining was used to assess pathological changes in the uterus, which were improved in the uterine muscle from mice in the $\mathrm{Rg} 1, \mathrm{Rg} 1+\mathrm{D}$-gal and saline groups. Red arrows indicate the location of the endometrium. Scale bar, $100 \mu \mathrm{m}$. (B) Statistical analysis of H\&E staining results ( $\mathrm{n}=3)$. $^{*} \mathrm{P}<0.05$ vs. D-gal. POI, premature ovarian insufficiency; Rg1, ginsenoside Rg1; D-gal, D-galactose.

A

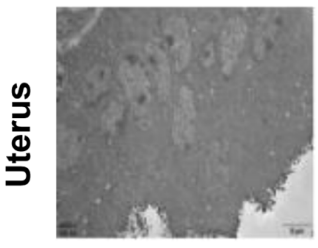

Saline

B

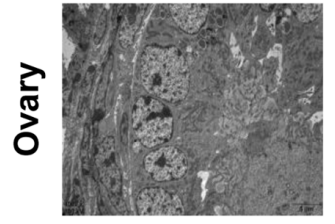

Saline

C

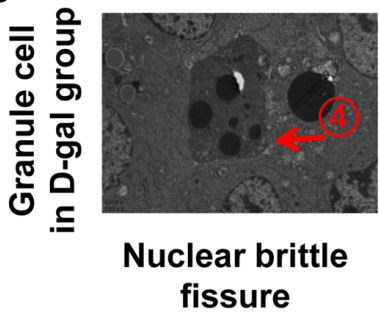

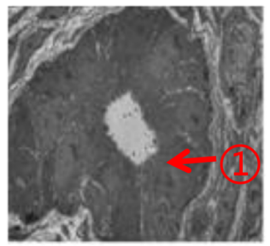

Rg1+D-gal

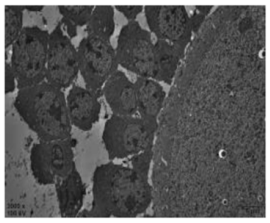

Rg1+D-gal

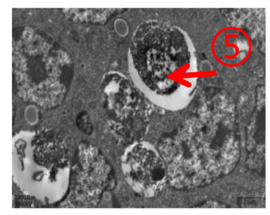

Nuclear dissolution

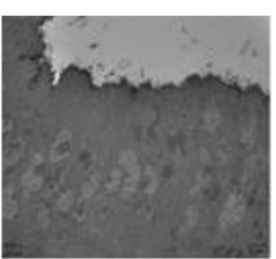

Rg1

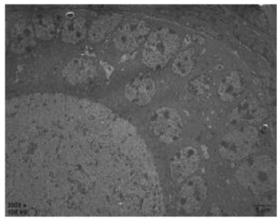

Rg1

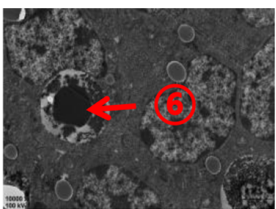

Nucleation

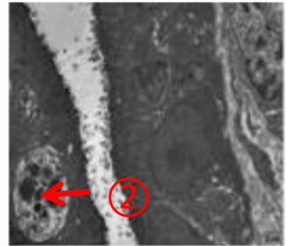

D-gal

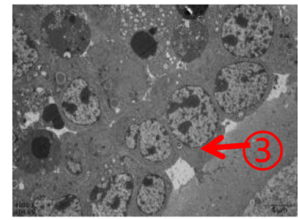

D-gal

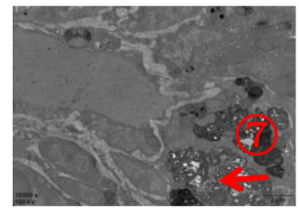

Necrotic tissue in ovarian stroma

Figure 3. Pathological changes in the uterus and ovary as evaluated using electron microscopy. (A) In the uterus, the endometrial glands were found in the Rg1 + D-gal group as indicated by arrow 1 and 2 shows the myeloid bodies in the granule cells. (B) There were also large amounts of myeloid bodies in the granule cells of the ovary, as indicated by red arrow 3. (C) In the granule cells of the D-gal group, arrow 4 indicates nuclear brittle fissure, arrow 5 shows nuclear dissolution and arrow 6 indicates nucleation. However, none of these features could be observed in the Rg1, Rg1 + D-gal or saline groups. Furthermore, there were large numbers of myeloid bodies and larger extent of fibrosis in the uterine muscle in the D-gal group, as indicated by arrow 7, which were improved in the uterine muscle of Rg1, Rg1 + D-gal and saline groups. Magnification, x4. Rg1, ginsenoside Rg1; D-gal, D-galactose.

that $\operatorname{Rg} 1$ can alleviate pathological damage in the ovary and uterus of mice following D-gal induced POI.

Rg1 treatment upregulates the levels of E2, FSH and AMH in serum. Hormone levels after Rg1 treatment in the POI models were next investigated. On day 42 of administration, there were significantly lower AMH and E2 levels and significantly higher FSH levels in the D-gal group compared with those in the other three groups (Fig. 4). By contrast, Rg1 + D-gal group exhibited comparable levels of these hormones compared with those in the saline and $\operatorname{Rg} 1$ groups (Fig. 4). This observation suggests that $\mathrm{Rg} 1$ serves an anti-aging role in this D-gal-induced POI mouse model.

Rgl improves the ovarian damage induced by D-gal. The role of $\mathrm{Rg} 1$ on ovarian follicle maturation was examined further using $H \& E$ staining. The numbers of primary, secondary, sinus follicles and corpus luteum (CL) in the Rg1 + D-gal group were higher compared with those in the D-gal group (Fig. 5). At day 42 after D-gal administration, 
A

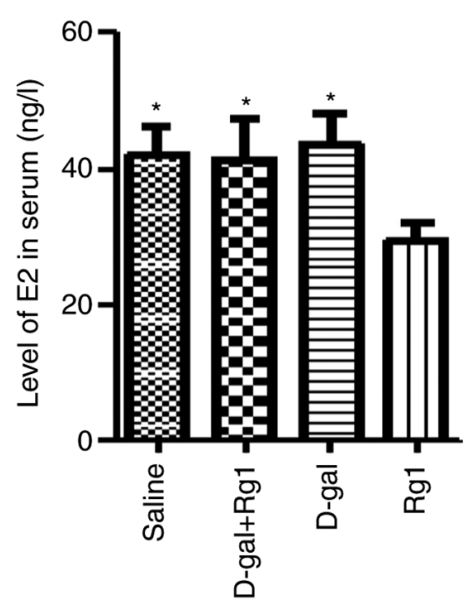

B

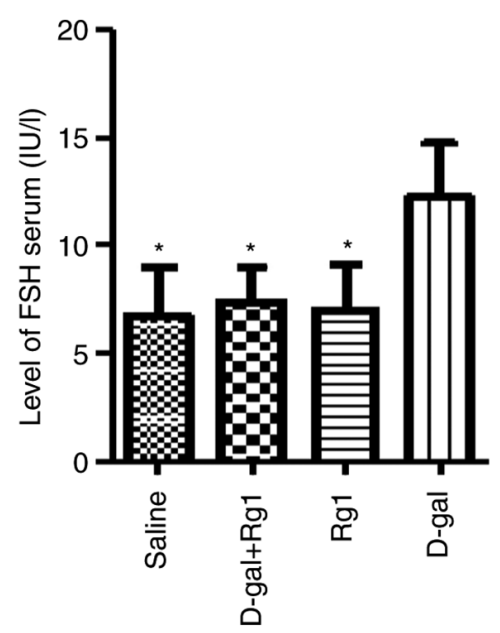

C

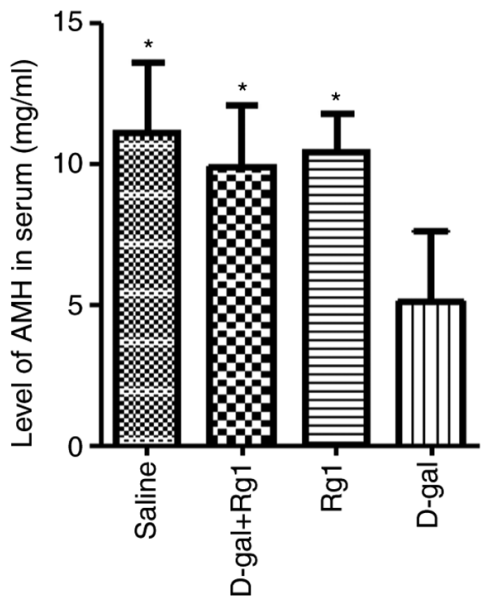

Figure 4. ELISA analysis of E2, FSH and AMH. On day 42, serum levels of (A) E2, (B) FSH and (C) AMH were measured using ELISA. "P<0.05 vs. D-gal. Rg1, ginsenoside Rg1; D-gal, D-galactose; E2, estradiol 2; FSH, follicle-stimulating hormone; AMH, anti-mullerian hormone.

A

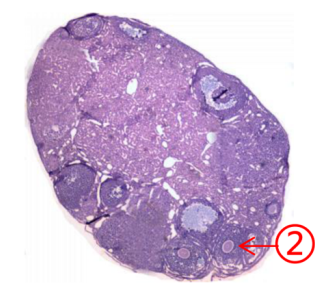

Saline

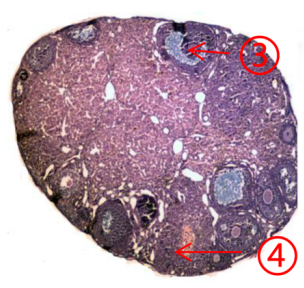

Rg1

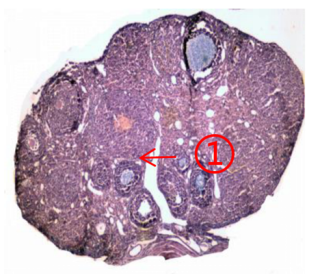

Rg1+D-gal

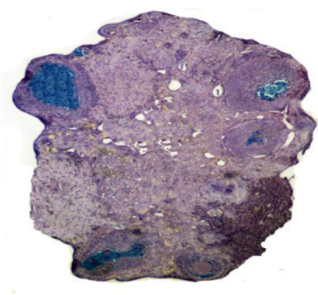

D-gal
B

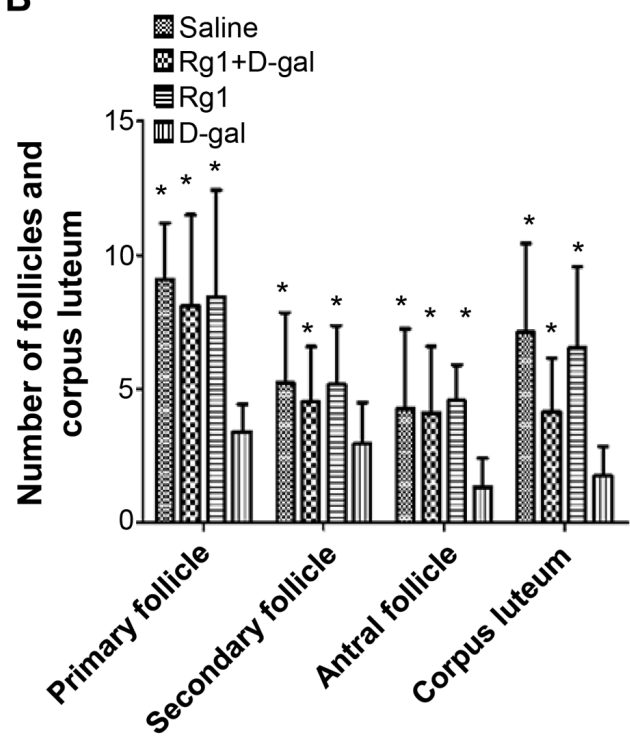

Figure 5. H\&E staining of ovarian follicles. On day 42 , ovaries were harvested and subjected to the H\&E staining. (A) Representative H\&E images (magnification, $\mathrm{x} 4$ ). Arrow 1 indicates the primary follicle, arrow 2 indicates the secondary follicle, arrow 3 indicates the sinus follicle and arrow 4 indicates the location of corpus luteum. (B) Follicle numbers were then counted. "P<0.05 vs. D-gal. Rg1, ginsenoside Rg1; D-gal, D-galactose.

Rg1, Rg1 + D-gal and saline groups exhibited follicles of different stages and CL. However, a distinct ovary architecture was observed in the D-gal group, where the ovary mass was reduced and there were no small follicles, CL or antral follicles (Fig. 5A). In addition, ovaries from the D-gal + Rg1 group exhibited significantly more $\mathrm{CL}$, antral follicles and growing follicles compared with those in the D-gal group (Fig. 5B). Ovaries from the saline, $\operatorname{Rg} 1$ and $\mathrm{Rg} 1+\mathrm{D}$-gal groups also demonstrated similar follicle numbers without significant differences among each other (Fig. 5B). These results suggest that $\mathrm{Rg} 1$ can improve the ovarian damages induced by D-gal treatment in POI mice.

Anti-oxidative effects of Rgl on ovary and uterus of POI mice. The anti-oxidative effects of Rg1 were next evaluated by measuring GSH-Px activity, MDA content and T-SOD activity in the ovary and uterus. Compared with those in the saline group, tissues from the D-gal group had significantly reduced GSH-Px and T-SOD activities, but significantly increased MDA content (Fig. 6). However, these effects aforementioned were found to be significantly reversed by Rg1 (Fig. 6). These results suggest that $\operatorname{Rg} 1$ exerts anti-oxidative effects by enhancing the activities of endogenous anti-oxidative defense enzymes.

Rg1 decreases pro-inflammatory cytokines levels in POI mice. Chronic inflammation in the ovary and uterus is associated with POI (4). Therefore, TNF- $\alpha$, IL-6 and IL- $1 \beta$ levels in the ovary and uterus tissues were subsequently measured using ELISA. Tissues from the D-gal + Rg1 group exhibited significantly reduced TNF- $\alpha$, IL- 6 and IL-1 $\beta$ levels compared with those in the D-gal group (Fig. 7). This suggests that $\operatorname{Rg} 1$ 
A

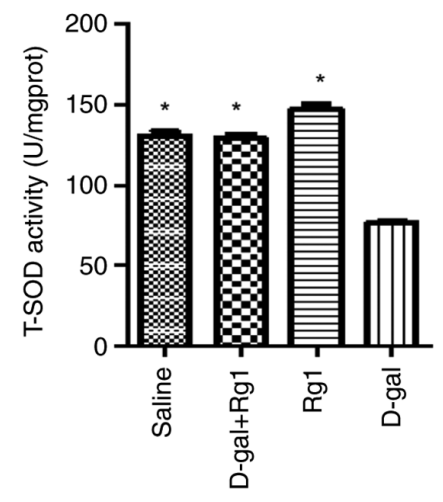

B

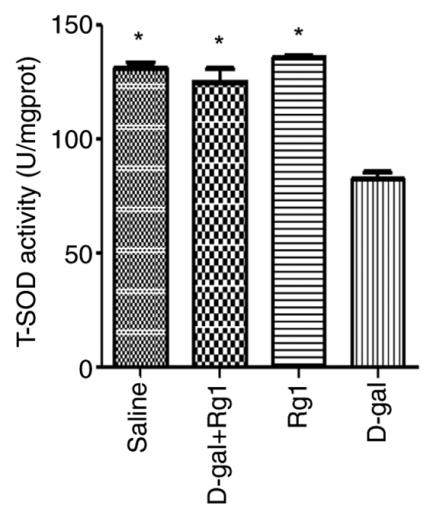

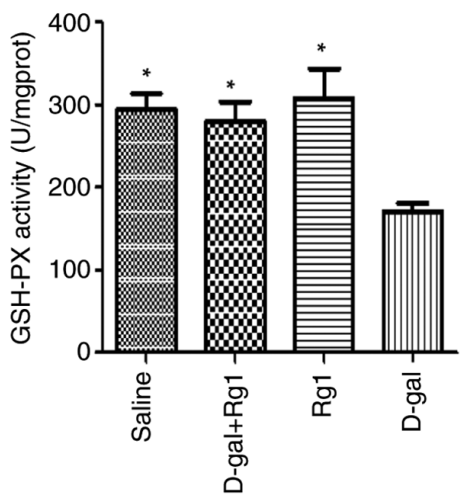

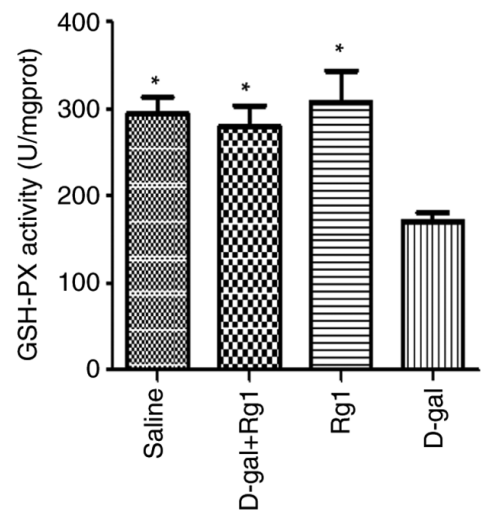

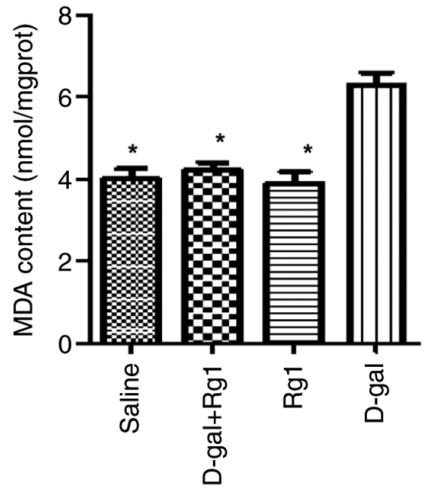

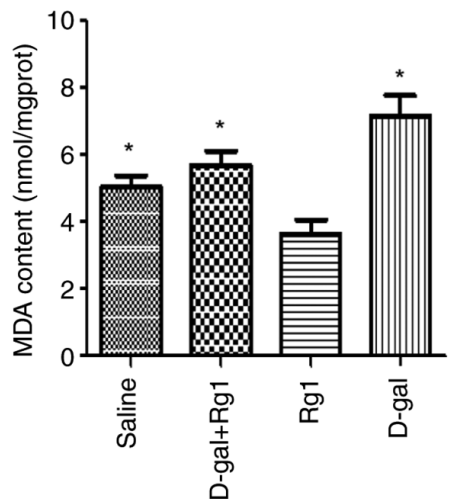

Figure 6. Effects of Rg1 treatment on the expression of oxidative stress markers and the activities of antioxidants in the uterus and ovary. On day 42, T-SOD activity, MDA contents, and GSH-Px activity were evaluated by chemical colorimetric analysis in the (A) ovaries and (B) uterus. "P<0.05 vs. D-gal. T-SOD, total superoxide dismutase; MDA, malondialdehyde; GSH-Px, glutathione peroxidase; Rg1, ginsenoside Rg1; D-gal, D-galactose.
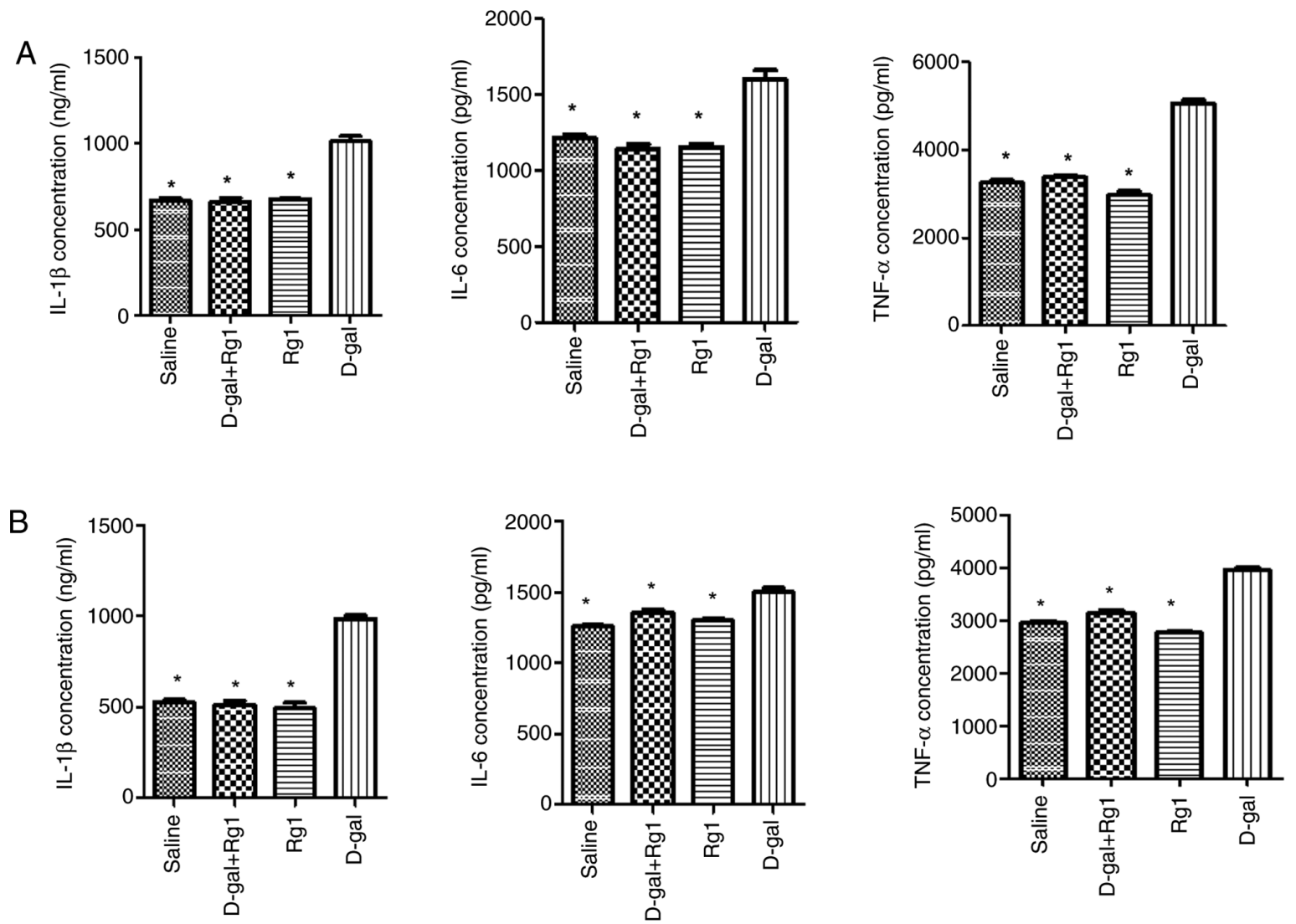

Figure 7. Effects of $\operatorname{Rg} 1$ on the expression of inflammatory indicators in the uterus and ovary. On day 42 , the levels of IL-1 $\beta$, IL- 6 and TNF- $\alpha$ in the (A) ovaries and (B) uterus were evaluated by ELISA. "P<0.05 vs. D-gal. IL, interleukin; TNF- $\alpha$, tumor necrosis factor- $\alpha$; Rg1, ginsenoside Rg1; D-gal, D-galactose. 

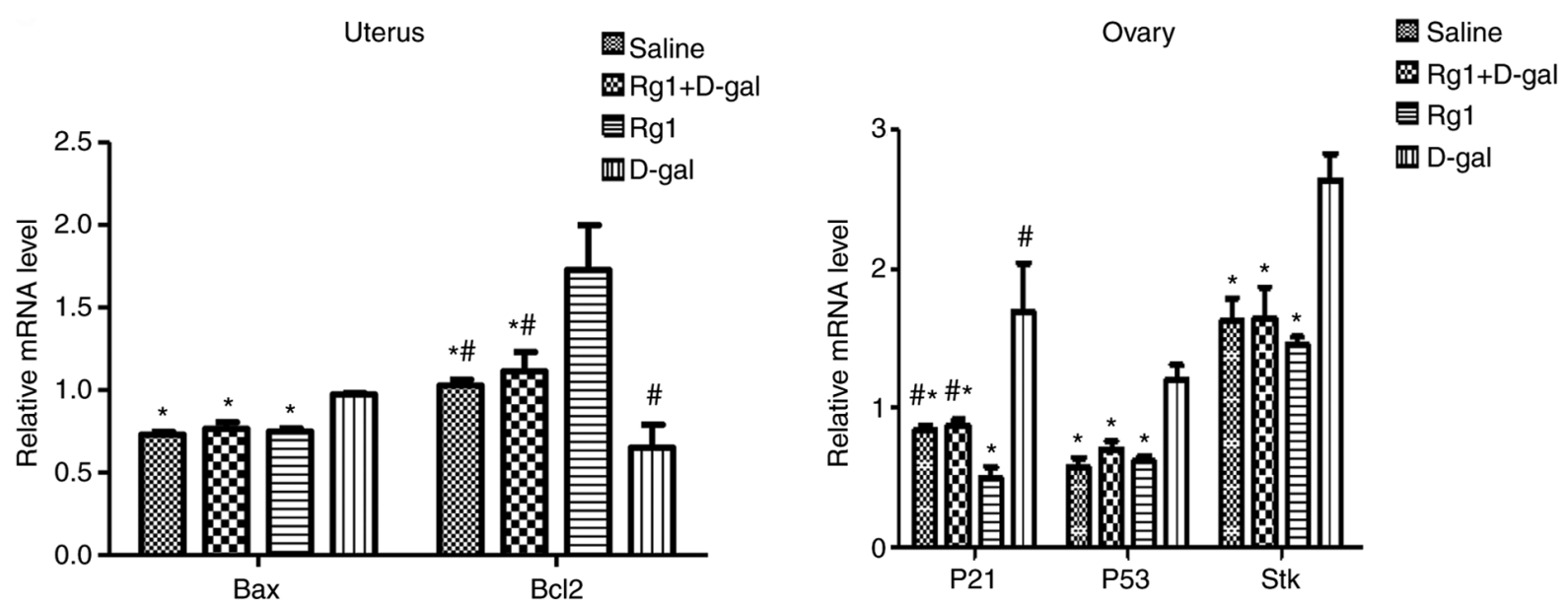

Figure 8. Effects of Rg1 on the expression of key components of the senescence pathway in the uterus and ovary. Relative mRNA relative expression levels of Bax, Bcl-2, p53, p21 and STK were measured in the uterus and ovaries using reverse transcription-quantitative PCR. "P<0.05 vs. D-gal and ${ }^{\text {"} P}<0.05$ vs. Rg1. Rg1, ginsenoside Rg1; D-gal, D-galactose; STK, serine/threonine kinase.

treatment alleviates inflammation in D-gal-induced aging in the ovary and uterus.

Rgl affects aging-related signaling pathways in ovary and uterus. To determine whether Rg1 could affect the signaling pathways related to aging in the uterus (Bax-Bcl-2) and ovary (p53-p21-STK) (34,35), RT-qPCR was performed. The relative mRNA expression levels of Bax in the uterus, and p53, p21 and STK in the ovary tissues of the D-gal group were significantly higher compared with those in the Rg1 group (Fig. 8). By contrast, the relative mRNA expression levels of $\mathrm{Bcl}-2$ in the uterus of the D-gal group were significantly lower compared with those in the Rg1 group (Fig. 8). These results suggest that $\mathrm{Rg} 1$ can reverse the changes in the relative mRNA expression of Bax, p53, p21, STK and Bcl-2 induced by D-gal in POI mouse models.

\section{Discussion}

Aging is the phenomenon of degenerative changes in the structure and function of human cells and tissues, which is an inevitable biological process (36). The main manifestations of this process include organ weight loss, cell atrophy, cytoplasmic pigmentation, reduced functional metabolism, weakened adaptive capacity and low disease resistance (37). Investigation on aging has become a research hotspot (38). POI represents a spectrum of disorders in the female reproductive system that leads to ovarian dysfunction (39). Its clinical criteria primarily include: i) Secondary amenorrhea for $\geq 4$ consecutive months; ii) elevated FSH level (FSH $>25 \mathrm{U} / 1$, for two times, with the time interval $>4$ weeks); and iii) low estradiol levels before the age of 40 years (40). POI can result in senile dementia, osteoporosis and climacteric syndrome in female individuals, seriously affecting their health (41). It eventually develops into premature ovarian failure $(\mathrm{FSH}>40 \mathrm{U} / \mathrm{l})$ and the decreased estrogen levels, which is accompanied by varying degrees of perimenopausal symptoms and represents the end stage of POI (42). However, its pathogenesis remains unclear.

Ginsenoside Rg1 can be isolated from Panax ginseng and has been used in traditional Chinese medicine for $>2,000$ years.
$\mathrm{Rg} 1$ has been documented to confer a range of effects on aging rats and mice, including increasing mass index of uterus and ovary, amelioration of perimenopausal syndromes, recovery of estrous cycle, and promotion of hormone secretion $(43,44)$. In addition, $\mathrm{Rg} 1$ also exhibits antioxidant and proliferation-promoting activities (43). Except for ovary and uterus, D-gal administration has been previously demonstrated to cause inflammatory damage to the liver, lungs and spleen (43). D-gal aging model was previously established (45). In the present study, this model was used to investigate the potential anti-aging effects of Rg-1 on POI. Following treatment with D-gal, high quantities of myeloid bodies, nuclear brittle fissure, nuclear dissolution and nucleation were observed in the granule cells. This was also coupled with the observation of necrotic tissue in the ovarian stroma. In addition, myeloid bodies and fibrosis were observed in the uterine muscle of the D-gal group after administration of D-gal for 42 days, which was attenuated by Rg1 treatment. By contrast, D-gal treatment induced serious and persistent damage in the ovaries. Additionally, D-gal significantly decreased the secretion of sex hormones and the numbers of ovarian follicles. These results suggest that D-gal treatment induced ovary failure and reduced ovary function in female mice. However, Rg1 administration increased the weights of ovary and uterus in D-gal-treated mice, demonstrating potential functional recovery in the ovary and uterus caused by Rg1. Rg1 was also found to delay ovary granular cell senescence (Fig. 3) and prevented endometrium destruction by mediating anti-oxidant and anti-inflammatory effects. AMH can be used as one of the biomarkers for assessing ovary function (46), whereas follicle counts can be applied to reflect ovary reservation (47). In addition, endocrine function can be reflected mainly by measuring hormone levels, including FSH, LH and E2 (48).

Based on the provocative tests, dysfunctional gonads may be observed in $75-96 \%$ of patients with galactosemia, which may result in hypergonadotropic hypogonadism (43). This can be explained by previous findings that galactose can attenuate FSH bioactivity and exert direct toxic effects (49). In addition, FSH can be used to indirectly reflect hypothalamic response and ovary function (50). AMH is secreted by primary 
granulosa cells through the preovulatory follicles during the late preantral stage (51) that can be used to reflect ovary function during classic galactosemia (52). It was found in the present study that in mice treated with D-gal, serum FSH levels were significantly increased, whilst the serum AMH and E2 levels were significantly reduced in a manner that could be reversed by $\mathrm{Rg} 1$. Therefore, this suggests that $\mathrm{Rg} 1$ can delay senescence induced by D-gal.

Macroscopic ovary morphology parallels that of FSH levels (53). Qin et al (54) previously demonstrated that the number of oocytes was significantly decreased in female rats after exposure to high levels of galactose during gestation. Thakur et al (55) demonstrated that galactitol levels may affect follicular maturation and ovulation. In the present study, D-gal treatment decreased follicle numbers, which was reversed by Rg1. Serum FSH levels tend to be higher during follicle development failure in patients with POI patients, suggesting that FSH receptor function may be either attenuated or impaired on granule cells, causing insensitivity to FSH stimulation and resulting in inhibition of follicle growth (56).

Although the mechanism of aging is complex, the free oxygen radical theory has gained increasing attention (57). Under physiological conditions, oxygen free radicals produced in the body are in maintained in balance by the free radical scavenging system (58). When this balance is broken, the activities of antioxidant enzymes (SOD) is reduced (59). Excessive generation of oxygen free radicals would result in oxidative stress damage, leading to the increased production of the lipid peroxide MDA and eventually cell membrane damage, decreased tissue and organ function and subsequent aging of the body (60). Therefore, eliminating excess oxygen free radicals may serve to be an important strategy to delay aging. Accumulation of galactitol during galactosemia impairs free radical scavenging activities by interfering with glutathione reductase (23). Therefore, antioxidant therapy may minimize ovary and uterus damage in patients with galactosemia. In the present study, Rg1 treatment increased T-SOD and GSH-Px activities whilst decreasing MDA content. This suggests that Rg1 can protect the ovary against D-gal-induced oxidative stress, possibly by savaging free radicals and activating antioxidant enzymes. Notably, T-SOD activities were found to be increased in the mice treated only with $\mathrm{Rg} 1$ compared with the D-gal group, in line with previous studies (61-63), further confirming the antioxidative effects of Rg1. Oocyte apoptosis can be promoted by a number of factors, including Fas ligands, pro-inflammatory cytokines, gonadal $\mathrm{GnRH}$-like proteins and androgens (64). In the present study, Rg1 treatment inhibited the expression of pro-inflammatory cytokines TNF- $\alpha$, IL-6 and IL-1 $\beta$, suggesting that it exerted anti-inflammatory and oocyte-promoting effects.

p53 regulates $\mathrm{p} 21$ by modulating the expression of the downstream gene $P 21$, which is a cyclin-dependent kinase inhibitor (24). The $\mathrm{p} 21$ protein can in turn bind to the Cyclin-cdk complex, thereby inhibiting its protein kinase activity towards the retinoblastoma protein (65). Dephosphorylated $\mathrm{Rb}$ remains bound to the transcriptional regulator E2F, preventing it from activation, causing $\mathrm{G}_{1}$ arrest and cell senescence (66). A previous study has shown that the intrinsic death pathway mediated by p53 can induce follicular atresia (67), where p21 could be transcriptionally activated by this accumulation of the p53 protein $(68,69)$. The present study found that D-gal increased STK, p21 and p53 levels in a manner that was reversed by Rg1, suggesting that $\mathrm{Rg} 1$ can delay ovary aging by downregulating the expression of senescence markers. The pro-apoptotic protein Bax and anti-apoptotic protein Bcl-2 are common markers of apoptosis (70). Hussein et al (71) previously found that the Bcl-2 family member-mediated intrinsic pathway was the main mechanism underlying uterine cellular apoptosis. Bcl2-Bax operates antagonistically, which work by forming homodimers or heterodimers (72). When this balance in their expression is disrupted, they can form Bcl2-Bcl2, Bax-Bax homodimers or even Bcl2-Bax heterodimers. If Bax levels are high, the levels of Bax-Bax homodimers are increased, whilst Bcl2 activity would be decreased to accelerate apoptosis (73). By contrast, high levels of $\mathrm{Bcl} 2$ would lead to the formation of Bcl2-Bax heterodimers, which are more stable compared with homodimers and can inhibit apoptosis (74). The Bcl2/Bax ratio serves key roles in ovarian granulocyte apoptosis (75). The present study revealed that D-gal increased Bax expression whilst decreasing that of $\mathrm{Bcl}-2$ to promote apoptosis but Rg1 treatment inhibited apoptosis by upregulating Bax and whilst downregulating Bcl-2, which delayed ovarian aging caused by D-gal.

In conclusion, $\operatorname{Rg} 1$ was found to improve ovary and uterus pathological damage in POI mouse models by promoting their antioxidant and anti-inflammatory capacities. The underlying molecular mechanism(s) may be associated with the activation of the p21-p53-STK signaling pathway in the ovary and Bax-bcl2 in the uterus.

\section{Acknowledgements}

Not applicable.

\section{Funding}

The present study was supported by the National Natural Science Foundation of China Grants (grant no. X2271), the Guizhou Provincial Administration of traditional Chinese Medicine and Science and Technology Research of traditional Chinese Medicine (grant no. QZYY-2019-093) and the Science and Technology Project Jointly Supported by the Zunyi City and The First People's Hospital of Zunyi (grant no. 187; 2018).

\section{Availability of data and materials}

The datasets used and/or analyzed during the current study are available from the corresponding author on reasonable request.

\section{Authors' contributions}

ZX, LH and XL designed the study. LH provided the theoretical knowledge of POI, collected the experimental data, performed the H\&E staining, and the electron microscopy experiments. $\mathrm{XW}$ assisted in the completion of electron microscopy experiments and ELISA. DC performed the PCR assay, and collected the experimental data. $\mathrm{LH}, \mathrm{ZX}$ and XL interpreted the data. DC searched the literature. LH prepared the manuscript. All authors read and approved the final manuscript. 


\section{Ethics approval and consent to participate}

All animal experimental procedures were performed in accordance with the Institutional Animal Care and Use Committee of Chongqing Medical University, which was approved by the Institutional Animal Care and Use Committee of Chongqing Medical University (Chongqing, China).

\section{Patient consent for publication}

Not applicable.

\section{Competing interests}

The authors declare that they have no competing interests.

\section{References}

1. Rossetti R, Ferrari I, Bonomi M and Persani L: Genetics of primary ovarian insufficiency. Clin Genet 91: 183-198, 2017.

2. Laven JS: Primary ovarian insufficiency. Semin Reprod Med 34: 230-234, 2016.

3. Qin C, Chen Y, Lin Q, Yao J, Wu W and Xie J: The significance of polymorphism and expression of oestrogen metabolism-related genes in Chinese women with premature ovarian insufficiency. Reprod Biomed Online 35: 609-615, 2017.

4. Huang Y, Hu C, Ye H, Luo R, Fu X, Li X, Huang J, Chen W and Zheng Y: Inflamm-aging: A new mechanism affecting premature ovarian insufficiency. J Immunol Res 2019: 8069898, 2019.

5. European Society for Human Reproduction and Embryology (ESHRE) Guideline Group on POI; Webber L, Davies M Anderson R, Bartlett J, Braat D, Cartwright B, Cifkova R, de Muinck Keizer-Schrama S, Hogervorst E, et al: ESHRE guideline: Management of women with premature ovarian insufficiency. Hum Reprod 31: 926-937, 2016.

6. Soman M, Huang LC, Cai WH, Xu JB, Chen JY, He RK, Ruan HC, Xu XR, Qian ZD and Zhu XM: Serum androgen profiles in women with premature ovarian insufficiency: A systematic review and meta-analysis. Menopause 26: 78-93, 2019.

7. Vujović S, Ivovic M, Tančić-Gajić M, Marina L, Ljubic A, Dragojević-Dikić S and Genazzani AR: Endometrium receptivity in premature ovarian insufficiency-how to improve fertility rate and predict diseases?. Gynecol Endocrinol 34: 1011-1015, 2018.

8. Patel H, Arruarana V, Yao L, Cui X and Ray E. Effects of hormones and hormone therapy on breast tissue in transgender patients: A concise review. Endocrine 68: 6-15, 2020.

9. Zhu C, Wang Y, Liu $\mathrm{H}, \mathrm{Mu} \mathrm{H}$, Lu Y, Zhang J and Huang J: Oral administration of Ginsenoside Rg1 prevents cardiac toxicity induced by doxorubicin in mice through anti-apoptosis Oncotarget 8: 83792-83801, 2017.

10. Zhu J, Mu X, Zeng J, Xu C, Liu J, Zhang M, Li C, Chen J, Li T and Wang Y: Ginsenoside Rg1 prevents cognitive impairment and hippocampus senescence in a rat model of D-galactose-induced aging. PLoS One 9: e101291, 2014.

11. Yang N, Zhou B, Wang Y, Li Y, Liu J, Li P, Yang N, Zhou B, Wang Y et al: Research progress of the biological activity of the ginsenoside Rg. Chinese J Traditional Chinese Med 33 : 1463-1465, 2018 (In Chinese).

12. Tang F, Lu M, Yu L, Wang Q, Mei M, Xu C, Han R, Hu J, Wang H and Zhang Y: Inhibition of TNF- $\alpha$-mediated NF- $\kappa$ B activation by ginsenoside $\mathrm{Rg} 1$ contributes the attenuation of cardiac hypertrophy induced by abdominal aorta coarctation. J Cardiovasc Pharmacol 68: 257-264, 2016.

13. Lee HY, Park SH, Chae SW, Soung NK, Oh MJ, Kim JS, Kim YO and Chae HJ: Aqueous ginseng extract has a preventive role in RANKL-induced osteoclast differentiation and estrogen deficiency-induced osteoporosis. J Functional Foods 13: 192-203, 2015.

14. Cui Y, Shu XO, Gao YT, Cai H, Tao MH and Zheng W: Association of ginseng use with survival and quality of life among breast cancer patients. Am J Epidemiol 163: 645-653, 2006.

15. Ryan J, Scali J, Carriere I, Ritchie K and Ancelin ML: Hormonal treatment, mild cognitive impairment and Alzheimer's disease. Int Psychogeriatr 20: 47-56, 2008.
16. Zhao $\mathrm{E}$ and $\mathrm{Mu}$ Q: Phytoestrogen biological actions on Mammalian reproductive system and cancer growth. Sci Pharm 79: 1-20, 2011.

17. Cheng Y, Shen LH and Zhang JT: Anti-amnestic and anti-aging effects of ginsenoside Rg1 and Rb1 and its mechanism of action. Acta Pharmacol Sin 26: 143-149, 2005.

18. Li J, Cai D, Yao X, Zhang Y, Chen L, Jing P, Wang L and Wang Y: Protective effect of ginsenoside Rg1 on hematopoietic stem/progenitor cells through attenuating oxidative stress and the Wnt/ $\beta$-Catenin signaling pathway in a mouse model of d-Galactose-induced aging. Int J Mol Sci 17: 849, 2016.

19. He L: Establishment of D-gal induced premature ovarian failure animal model. Proc Clin Med 25: 762-764, 2016. (In Chinese)

20. Huang JL, Yu C, Su M, Yang SM, Zhang F, Chen YY, Liu JY, Jiang YF, Zhong ZG et al: Probucol, a "non-statin" cholesterollowering drug, ameliorates D-galactose induced cognitive deficits by alleviating oxidative stress via Keap1/Nrf2 signaling pathway in mice. Aging (Albany NY) 11: 8542-8555, 2019.

21. Thakur M, Shaeib F, Khan SN, Kohan-Ghadr HR, Jeelani R, Aldhaheri SR, Gonik B and Abu-Soud HM: Galactose and its metabolites deteriorate metaphase II mouse oocyte quality and subsequent embryo development by disrupting the spindle structure. Sci Rep 7: 231, 2017

22. Banerjee S, Chakraborty P, Saha P,Bandyopadhyay SA, Banerjee S and Kabir SN: Ovotoxic effects of galactose involve attenuation of follicle-stimulating hormone bioactivity and up-regulation of granulosa cell p53 expression. PLoS One 7: e30709, 2012.

23. Sozen B, Ozekinci M, Erman M, Gunduz T, Demir N and Akouri R: Dehydroepiandrosterone supplementation attenuates ovarian ageing in a galactose-induced primary ovarian insufficiency rat model. J Assist Reprod Genet 36: 2181-2189, 2019.

24. Kim EM, Jung CH, Kim J, Hwang SG, Park JK and Um HD: The p53/p21 complex regulates cancer cell invasion and apoptosis by targeting Bcl-2 family proteins. Cancer Res 77: 3092-3100, 2017.

25. Zińczuk J, Zaręba K, Guzińska-Ustymowicz K, Kędra B, Kemona A and Pryczynicz A: p16, p21, and p53 proteins play an important role in development of pancreatic intraepithelial neoplastic. Ir J Med Sci 187: 629-637, 2018.

26. Ibnat N, Kamaruzman NI, Ashaie M and Chowdhury EH: Transfection with p21 and p53 tumor suppressor plasmids suppressed breast tumor growth in syngeneic mouse model. Gene 701: 32-40, 2019.

27. Fujino T, Yokokawa R, Oshima T and Hayakawa M: SIRT knockdown up-regulates p53 and p21/Cip1 expression in renal adenocarcinoma cells but not in normal renal-derived cells in a deacetylase-independent manner. J Toxicol Sci 43: 711-715, 2018.

28. Dmitrieva AI, Serebryakova VA, Rakitin SS, Kudyakov LA, Novitskii VV, Yankovich KI and Sevostyanova NV: Study of the association of polymorphisms of p53 and p21 with the risk of development of stomach cancer. Bull Exp Biol Med 164: 95-98, 2017.

29. Ramadan MA, Shawkey AE, Rabeh MA and Abdellatif AO: Expression of P53, BAX, and BCL-2 in human malignant melanoma and squamous cell carcinoma cells after tea tree oil treatment in vitro. Cytotechnology 71: 461-473, 2019.

30. Cicero L, Fazzotta S, Palumbo VD, Cassata G and Lo Monte AI Anesthesia protocols in laboratory animals used for scientific purposes. Acta Biomed 89: 337-342, 2018.

31. Boivin GP, Bottomley MA, Schiml PA, Goss L and Grobe N: Physiologic, behavioral, and histologic responses to various euthanasia methods in C57BL/6NTac male mice. J Am Assoc Lab Anim Sci 56: 69-78, 2017.

32. Wang Q, Yin J, Wang S, Cui D, Lin H, Ge M, Dai Z, Xie L, Si J, Ma K, et al: Effects of activin A and its downstream ERK1/2 in oxygen and glucose deprivation after isoflurane-induced postconditioning. Biomed Pharmacother 84: 535-543, 2016.

33. Livak KJ and Schmittgen TD: Analysis of relative gene expression data using real-time quantitative PCR and the 2(-Delta Delta C(T)) method. Methods 25: 402-408, 2001.

34. Saat N, Risvanli A, Dogan H, Onalan E, Akpolat N, Seker I and Sahna E: Effect of melatonin on torsion and reperfusion induced pathogenesis of rat uterus. Biotech Histochem 94: 533-539, 2019.

35. Chen MJ, Chou CH, Shun CT, Mao TL, Wen WF, Chen CD, Chen SU, Yang YS and Ho HN: Iron suppresses ovarian granulosa cell proliferation and arrests cell cycle through regulating p38 mitogen-activated protein kinase/p53/p21 pathway. Biol Reprod 97: 438-448, 2017.

36. da Costa JP, Vitorino R, Silva GM, Vogel C, Duarte AC and Rocha-Santos T: A synopsis on aging-theories, mechanisms and future prospects. Ageing Res Rev 29: 90-112, 2016. 
37. Flatt $\mathrm{T}$ and Partridge L: Horizons in the evolution of aging. BMC Biol 16: 93, 2018.

38. Sun C, Fan S, Wang X, Lu J, Zhang Z, Wu D, Shan Q and Zheng Y: Purple sweet potato color inhibits endothelial premature senescence by blocking the NLRP3 inflammasome. J Nutr Biochem 26: 1029-1040, 2015.

39. Sullivan SD, Sarrel PM and Nelson LM: Hormone replacement therapy in young women with primary ovarian insufficiency and early menopause. Fertil Steril 106: 1588-1599, 2016.

40. Riegle van West K, Stinear C and Buck R: The effects of poi on physical and cognitive function in healthy older adults. J Aging Phys Act, Nov 82018 (Online ahead of print).

41. Sundaresan NR, Saxena VK, Sastry KV, Nagarajan K, Jain P, Singh R, Anish D, Ravindra PV, Saxena M and Ahmed KA: Cytokines and chemokines in postovulatory follicle regression of domestic chicken (Gallus gallus domesticus). Dev Comp Immunol 32: 253-264, 2008.

42. He L, Ling L, Wei T, Wang Y and Xiong Z: Ginsenoside Rg1 improves fertility and reduces ovarian pathological damages in premature ovarian failure model of mice. Exp Biol Med (Maywood) 242: 683-691, 2017.

43. Mou Z, Huang Q, Chu SF, Zhang MJ, Hu JF, Chen NH and Zhang JT: Antidepressive effects of ginsenoside Rg1 via regulation of HPA and HPG axis. Biomed Pharmacother 92: 962-971, 2017.

44. Mo J, Zhou Y, Yang R, Zhang P, He B, Yang J, Li S, Shen Z and Chen P: Ginsenoside Rg1 ameliorates palmitic acid-induced insulin resistance in HepG2 cells in association with modulating Akt and JNK activity. Pharmacol Rep 71: 1160-1167, 2019.

45. Yan Z, Dai Y, Fu H, Zheng Y, Bao D, Yin Y, Chen Q, Nie X, Hao Q, Hou D and Cui Y: Curcumin exerts a protective effect against premature ovarian failure in mice. J Mol Endocrinol 60: 261-271, 2018.

46. Mullen RD, Ontiveros AE, Moses MM and Behringer RR: AMH and AMHR2 mutations: A spectrum of reproductive phenotypes across vertebrate species. Dev Biol 455: 1-9, 2019.

47. Roness H and Meirow D: Fertility preservation: Follicle reserve loss in ovarian tissue transplantation. Reproduction 158 F35-F44, 2019.

48. Warren L, Murawski M, Wilk K, Zieba DA and Bartlewski PM: Suitability of antral follicle counts and computer-assisted analysis of ultrasonographic and magnetic resonance images for estimating follicular reserve in porcine, ovine and bovine ovaries ex situ. Exp Biol Med (Maywood) 240: 576-584, 2015.

49. Song D, Zhong Y, Qian C, Zou Q, Ou J, Shi Y, Gao L, Wang G, Liu Z, Li H, et al: Human umbilical cord mesenchymal stem cells therapy in cyclophosphamide-induced premature ovarian failure rat model. Biomed Res Int 2016: 2517514, 2016.

50. Liu Y, Li YJ and Wang L: Changes of expression of p53-p21 in premature ovarian failure in fluorosis female mice. Chongqing Med 13: 1712-1715, 2018.

51. Podfigurna-Stopa A, Czyzyk A, Grymowicz M, Smolarczyk R, Katulski K, Czajkowski K and Meczekalski B: Premature ovarian insufficiency: The context of long-term effects. J Endocrinol Invest 39: 983-990, 2016

52. He L: Preliminary study on animal model of ovarian premature failure induced by d-galactose. Clin Med Practice 10: 762-764, 2016.

53. Ali DE, Shah M, Ali A, Malik MO, Rehman F, Badshah H, Ehtesham E and Vitale SG: Treatment with metformin and combination of metformin plus pioglitazone on serum levels of IL-6 and IL-8 in polycystic ovary syndrome: A randomized clinical trial. Horm Metab Res 51: 714-722, 2019.

54. Qin C, Yuan Z, Yao J, Zhu W, Wu W and Xie J: AMH and AMHR2 genetic variants in Chinese women with primary ovarian insufficiency and normal age at natural menopause. Reprod Biomed Online 3: 311-318, 2014.

55. Thakur M, Feldman G and Puscheck EE: Primary ovarian insufficiency in classic galactosemia: Current understanding and future research opportunities. J Assist Reprod Genet 35: 3-16, 2018.

56. Wang W, Wang Y, Wu J, Yang L and Liu Z: Protective effects of electroacupuncture pretreatment on ovarian in rats with premature ovarian insufficiency. Zhongguo Zhen Jiu 38: 405-411, 2018 (In Chinese)
57. Yuan S, Wen J, Cheng J, Shen W, Zhou S, Yan W, Shen L, Luo A and Wang S: Age-associated up-regulation of EGR1 promotes granulosa cell apoptosis during follicle atresia in mice through the NF- $\kappa B$ pathway. Cell Cycle 15: 2895-2905, 2016.

58. Yang F, Pei R, Zhang Z, Liao J, Yu W, Qiao N, Han Q, Li Y, $\mathrm{Hu} \mathrm{L}$, Guo J, et al: Copper induces oxidative stress and apoptosis through mitochondria-mediated pathway in chicken hepatocytes. Toxicol In Vitro 54: 310-316, 2019.

59. Falfushynska HI, Gnatyshyna LL, Deneha HV, Osadchuk OY and Stoliar OB: Manifestations of oxidative stress and molecular damages in ovarian cancer tissue. Ukr Biochem J 87: 93-102, 2015.

60. Zhang L, Kong XJ, Wang ZQ, Xu FS and Zhu YT: A study on neuroprotective effects of curcumin on the diabetic rat brain J Nutr Health Aging 20: 835-840, 2016.

61. Biswas P, Mukhopadhyay A, Kabir SN and Mukhopadhyay PK: High-protein diet ameliorates arsenic-induced oxidative stress and antagonizes uterine apoptosis in rats. Biol Trace Elem Res 192: 222-233, 2019.

62. Xie W, Zhou P, Sun Y, Meng X, Dai Z, Sun G and Sun X: Protective effects and target network analysis of ginsenoside $\mathrm{Rg} 1$ in cerebral ischemia and reperfusion injury: A comprehensive overview of experimental studies. Cells 7: 270, 2018.

63. Fan C, Song Q, Wang P, Li Y, Yang M and Yu SY: Neuroprotective effects of ginsenoside-Rg1 against depression-like behaviors via suppressing glial activation, synaptic deficits, and neuronal apoptosis in rats. Front Immunol 9: 2889, 2018.

64. Yang MZ, Li SG, et al: Effect of ginsenosideRg3 on ageing of vascular smooth muscle cells and itsm echanism. Heart BrainVessel Dis 17: 1079-1082, 2015.

65. Chen M, Zhang H, Zhang G, Zhong A, Ma Q, Kai J, Tong Y, Xie S, Wang Y, Zheng H, et al: Targeting TPX2 suppresses proliferation and promotes apoptosis via repression of the PI3k/AKT/P21 signaling pathway and activation of p53 pathway in breast cancer. Biochem Biophys Res Commun 507: 74-82, 2018.

66. Uxa S, Bernhart SH, Mages CFS, Fischer M, Kohler R, Hoffmann S, Stadler PF, Engeland K and Müller GA: DREAM and RB cooperate to induce gene repression and cell-cycle arrest in response to p53 activation. Nucleic Acids Res 47: 9087-9103, 2019.

67. Ma M, Chen XY, Li B and Li XT: Melatonin protects premature ovarian insufficiency induced by tripterygium glycosides: Role of SIRT1. Am J Transl Res 9: 1580-1602, 2017.

68. Hussein MR: Apoptosis in the ovary: Molecular mechanisms. Hum Reprod Update 11: 162-177, 2005.

69. el-Deiry WS, Tokino T, Velculescu VE, Levy DB, Parsons R, Trent JM, Lin D, Mercer WE, Kinzler KW and Vogelstein B: WAF1, a potential mediator of p53 tumor suppression. Cell 75: 817-825, 1993.

70. Harper JW, Adami GR, Wei N, Keyomarsi K and Elledge SJ: The p21 Cdk-interacting protein Cip1 is a potent inhibitor of G1 cyclin-dependent kinases. Cell 75: 805-816, 1993.

71. Hussein MR, Haemel AK and Wood GS: Apoptosis and melanoma: Molecular mechanisms. J Pathol 199: 275-288, 2003.

72. Hormozi M, Ghoreishi S and Baharvand P: Astaxanthin induces apoptosis and increases activity of antioxidant enzymes in LS-180 cells. Artif Cells Nanomed Biotechnol 47: 891-895, 2019.

73. Vartak SV, Iyer D, Santhoshkumar TR, Sharma S, Mishra A, Goldsmith G, Srivastava M, Srivastava S, Karki SS, Surolia A, et al: Novel BCL2 inhibitor, Disarib induces apoptosis by disruption of BCL2-BAK interaction. Biochem Pharmacol 131: 16-28, 2017.

74. Rahmani M, Nkwocha J, Hawkins E, Pei X, Parker RE, Kmieciak M, Leverson JD, Sampath D, Ferreira-Gonzalez A and Grant S: Cotargeting BCL-2 and PI3K induces BAX-dependent mitochondrial apoptosis in AML cells. Cancer Res 78: 3075-3086, 2018

75. Mousa AM, Al-Fadhli AS, Rao MS and Kilarkaje N: Gestational lead exposure induces developmental abnormalities and up-regulates apoptosis of fetal cerebellar cells in rats. Drug Chem Toxicol 38: 73-83, 2015.

This work is licensed under a Creative Commons Attribution-NonCommercial-NoDerivatives 4.0 International (CC BY-NC-ND 4.0) License. 